\title{
Validating the Impact of Experience Design to Foster Persuasive Interventions that Promote Energy Efficiency in Intelligent Work Environments
}

\author{
Ane Irizar-Arrieta \\ DeustoTech, University of Deusto \\ Bilbao \\ ane.irizar@deusto.es
}

\author{
Diego Casado-Mansilla \\ DeustoTech, University of Deusto \\ Bilbao \\ dcasado@deusto.es
}

\author{
Aiur Retegi \\ Deusto Design, University of Deusto \\ Bilbao \\ aiur.retegi@deusto.es
}

\begin{abstract}
Due to the scarcity of energy resources foreseen in the near future, it is necessary to address the energy efficiency in an imminent way. This framework serves as a motivation for this doctoral thesis, which aims at promoting awareness about the energy efficiency through interactive systems that use Experience Design as a key factor.

In order to develop effective elements, a people-centred perspective is used to validate the impact of the experience and of persuasive interventions which are focused on the diversity of users in the work environment. To this end, after reviewing the state of the art and analyzing the users and context of the project, it is proposed to address the awareness for the diversity through the creation of multidimensional and flexible user taxonomies. This categorization will be linked to persuasive interventions targeted in a personalized manner to each user profile.

The strategies generated will be translated into design guidelines that will be applied through technology, both in physical devices and digital tools. In these cases, the interfaces developed will be analysed and refined in order to link the proposed interactions with the design of concrete experiences based on people's needs. Finally, the systems developed (tangible and digital) will be evaluated over a long period of time to find the impact of the experience design. In addition, the aim is to measure the level of awareness of energy efficiency after prolonged interaction with the proposed tools, adherence to the system, maintenance of awareness and behaviour change over time.
\end{abstract}

\section{CCS CONCEPTS}

- Human-centered computing $\rightarrow$ User studies; User interface design; User centered design;

\section{KEYWORDS}

Interaction design, Experience Design, Human Computer Interaction, Persuasive Technology, Sustainable behaviour change

\section{ACM Reference Format:}

Ane Irizar-Arrieta, Diego Casado-Mansilla, and Aiur Retegi. 2018. Validating the Impact of Experience Design to Foster Persuasive Interventions that

Permission to make digital or hard copies of part or all of this work for personal or classroom use is granted without fee provided that copies are not made or distributed for profit or commercial advantage and that copies bear this notice and the full citation on the first page. Copyrights for third-party components of this work must be honored.

For all other uses, contact the owner/author(s).

Interacción 2018, September 12-14, 2018, Palma, Spain

(c) 2018 Copyright held by the owner/author(s).

ACM ISBN 978-1-4503-6491-1/18/09.

https://doi.org/10.1145/3233824.3233842
Promote Energy Efficiency in Intelligent Work Environments. In Interacción 2018: XIX International Conference on Human Computer Interaction, September 12-14, 2018, Palma, Spain. ACM, New York, NY, USA, 2 pages. https://doi.org/10.1145/3233824.3233842

\section{INTRODUCTION}

Energy efficiency is an issue whose relevance has increased due to the scarcity of resources expected in the near future. To address environmental sustainability, there are two main approaches in the field of computing: 1) increasing devices with automated processes and 2) change the user behavior w strategies implemented through technology. This research aims to influence people to raise their awareness of the need to maintain sustainable behaviours in the workplace. In private contexts, such as the home environment, the end user is responsible for the expenditure and awareness increases due to, among other factors, information on energy consumption and the assumed responsibility. In other contexts, such as public or shared spaces, responsibility is assumed by third parties, so in most cases, the awareness is diminished, making initiatives to promote energy efficiency more difficult and complex. In this way, through the awareness and learning that emerges from the interaction with technological devices, it is intended that the awareness and acquired behaviour be maintained over time and be extrapolated to other areas beyond the work environment.

To involve the user and to design effective systems for this purpose, it is essential to align these strategies with people's needs. However, due to the complexity of the individual, designing universal systems can lead to poor outcomes. Thus, it is essential to take into account the diversity of people in order to implement strategies that can be adapted to users effectively. For this purpose, technology can help us to influence behaviour by applying interaction strategies designed with the diversity of users in mind. In addition, by addressing heterogeneity, concrete interactions and experiences can be designed in a non-intrusive and pleasurable way for people, maximizing the impact of the message or intervention designed. This research is part of GreenSoul, an H2020 research project that aims to promote energy efficiency in European workplaces.

\section{STATE OF THE ART}

The literature, while exploring the diversity of users [1], does not yet propose flexible and multidimensional user characterizations that can be used as a solid basis.There are also approaches that foster the value of User-Centered Design (UCD) [7], although no studies have been found to validate its impact. In the area of the design of personalized experiences and their linkage to the interaction design, a promising study, [4], serves as a basis on which 
to advance the validation of the impact of the experience. Finally, long-term evaluation is another factor to which it is intended to contribute, since no studies have been found that propose this type of evaluation, as [2] points out in its review of the State of the Art.

\section{EXPERIENCE DESIGN FOR ENERGY EFFICIENCY}

To achieve energy efficiency in workspaces, persuasive systems will be designed to implement personalized interactions and experiences: on the one hand, digital tools such as mobile or web applications will be used; on the other, persuasive strategies will be implemented on tangible devices. One of the devices developed in this framework is the Interactive Coaster, a coaster that implements different persuasive strategies to make users aware of the energy consumed by the devices they use regularly. For this, the device has lights on the surface that are illuminated in colors associated with the semantics of a traffic light (red, green and yellow).

\subsection{Awareness for user diversity}

Through User-Centered Design (UCD), it is intended to analyze the needs and requirements of people, involving the user at all times. In order to address diversity, taking as a starting point the classification proposed by Lockton et al. [5] (based on behaviour) and by Petkov et al. [6] (value-based), taxonomies that can be adapted to the complexity of the individual through flexible and multidimensional systems will be designed.

To design awareness strategies to address diversity, it is necessary to link relevant persuasive interventions to the proposed taxonomies. For this, in addition to reviewing the literature, the different strategies will be associated with the different profiles. For each persuasion strategy a different function will be implemented in the different systems designed (physical device and digital application). These customized strategies will be applied giving the user freedom at all times to decide what type of information and feedback to receive. This first approach should be evaluated to refine and implement the proposal with complementary strategies validated by the users.

\subsection{Linking interactions and experiences}

With the aim of promoting the effectiveness of the designed interactions, the opinion of the users will be analyzed, defining, through the DCU, the qualities of the designed interactions. For this, it is proposed to use the vocabulary and method proposed by [3]. The next step is to analyse the experience generated by the interaction with the system, in order to discover its impact and link it to sustainability awareness. Taking into account the results obtained, the interactions will be refined in order to improve the strategies to obtain a specific experience linked to each type of user, thus increasing the satisfaction of people as well as the acceptance of the message emitted. With the purpose of measuring the impact of experience design on awareness and behaviour change, an experiment will be developed that includes long-term evaluation, with several months of both quantitative (measuring the energy consumption of users) and qualitative (through interviews, questionnaires and other methodologies) experimentation. This will help to discover adherence to the system and validate whether awareness and behaviour change are sustained over time.

\section{EXPECTED RESULTS}

After completion of the long-term evaluation, the experimental period will be terminated. Through the analysis of the results, the aim is to validate the impact of the design of experiences (addressing diversity) as a key factor in raising awareness of energy efficiency. This will be assessed by measuring the degree of awareness at the end of the experiment, as well as by examining the energy consumption of the users (although due to the limitations of the work context, the energy savings are expected to be residual).

\section{CONCLUSIONS}

This doctoral thesis seeks to promote energy efficiency by improving the experiences generated by persuasive technology. The intention is to redefine people's interactions with digital and/or tangible systems, allowing intelligent spaces to involve the user by encouraging efficient behaviour, bearing in mind their individuality and requirements. Through the design of personalised experiences, the objective is to improve people's relationships with everyday devices (both physical and digital) and the assimilation of the information provided by them, which, in addition to improving energy efficiency, facilitates and improves people's relationships with their immediate environment.

\section{ACKNOWLEDGEMENTS}

This work has been partially supported by the European Commission through the project H2020 - 696129 - GREENSOUL.

\section{REFERENCES}

[1] Aykut Coskun and Cigdem Erbugb. 2016. Exploring and communicating user diversity for behavioural change. 4 (2016), 1357--1374.

[2] Aykut Coskun, John Zimmerman, and Cigdem Erbug. 2015. Promoting sustainability through behavior change: A review. Design Studies 41 (2015), 183-204.

[3] Sarah Diefenbach, Eva Lenz, and Marc Hassenzahl. 2013. An interaction vocabulary. describing the how of interaction.. In CHI'13 Extended Abstracts on Human Factors in Computing Systems. ACM, 607-612.

[4] Eva Lenz, Marc Hassenzahl, and Sarah Diefenbach. 2017. Aesthetic interaction as fit between interaction attributes and experiential qualities. New Ideas in Psychology 47 (2017), 80-90.

[5] Dan Lockton, David Harrison, and Neville A Stanton. 2012. Models of the user: designers' perspectives on influencing sustainable behaviour. Fournal of Design Research 14 10, 1-2 (2012), 7-27.

[6] Petromil Petkov, Suparna Goswami, Felix Köbler, and Helmut Krcmar. 2012. Personalised eco-feedback as a design technique for motivating energy saving behaviour at home. In Proceedings of the 7th Nordic Conference on Human-Computer Interaction: Making Sense Through Design. ACM, 587-596.

[7] Renee Wever, Jasper Van Kuijk, and Casper Boks. 2008. User-centred design for sustainable behaviour. International journal of sustainable engineering 1, 1 (2008), $9-20$. 